\title{
La propiocepción como estrategia didáctica en la enseñanza de la pronunciación
}

\author{
VERÓNICA MORENO CAMPOS \\ Universidad de Castilla la Mancha \\ veronica.moreno@uclm.es
}

\begin{abstract}
Resumen: En este artículo se presenta una propuesta didáctica para la enseñanza y la mejora de la pronunciación de estudiantes de español. La propuesta se enmarca en el paradigma comunicativo y en los principios de la didáctica en enseñanza de las lenguas. La propuesta desarrolla un método de enseñanza de la pronunciación basado en el modelo de doble ruta psicolingüístico y para la intervención se apoya en el paradigma propioceptivo, entendido desde una perspectiva holística donde la percepción y la producción se engloban dentro del acto comunicativo. La propuesta comienza con una evaluación de las dificultades en pronunciación que guíe la elaboración del plan de enseñanza personalizado según las dificultades del estudiante y finaliza con una reevaluación para determinar si los objetivos se han conseguido.
\end{abstract}

Palabras clave: enseñanza de la pronunciación, ELE, didáctica, propiocepción

\section{Proprioception as a didactic strategy in pronunciation teaching}

Abstract: This article presents a didactic proposal for teaching and improving the pronunciation of Spanish students. The proposal is framed within the communicative paradigm and the principles of didactics in language teaching. The proposal develops a pronunciation teaching method based on the psycholinguistic double route model. The intervention is based on the proprioceptive paradigm, understood from a holistic perspective where perception and production are included within the communicative act. The proposal begins with an assessment of pronunciation difficulties to guide the development of a personalised teaching plan according to the student's difficulties and ends with a re-evaluation to determine whether the objectives have been achieved.

Key words: pronuntiation teaching, ELE, didactics, proprioceptive paradigm

\section{La enseñanza de la pronunciación: estado de la cuestión}

Cuando un estudiante se enfrenta al aprendizaje de una nueva lengua, sin duda una de las cuestiones que más preocupan es la pronunciación, pues una correcta articulación facilitará la inteligibilidad de su expresión oral. Sin embargo, diversos estudios (Cortés, 2002; Gospodaric, 2007; Otero Doval, 2013; Hidalgo Navarro, 2015; Sifrar, 2017) han señalado cómo la pronunciación —entendida en el sentido amplio recogido en El Diccionario de términos clave de ELE (2008), que abarca también el componente prosódico (acentuación, ritmo y entonación) - ocupa un lugar marginal en los manuales de enseñanza del español. Estos autores destacan que aun cuando la pronunciación ocupa una parte del temario estándar, en la mayoría de las ocasiones no se abarca en su totalidad, pues es frecuente encontrar que solo se proporcionan indicaciones sobre algún aspecto segmental o suprasegmental. 
Una de las razones aducidas para explicar la poca atención recibida por la pronunciación en comparación con otros aspectos del lenguaje ha sido la asunción del «prejuicio de la simplicidad»: al ser el español una lengua transparente se considera que la conversión grafema/fonema es directa y la arbitrariedad del sistema es la excepción (Poch-Olivé, 2009; Santamaría, 2010; Padilla, 2015). Esta simplicidad se ha asentado sobre la comparación con la opacidad de otras lenguas como pueda ser el caso de las germánicas. Sin embargo, autores como Llisterri (2003) y Gil (2007) han señalado la necesidad de una formación en fonética para poder enseñar pronunciación, y es que la aparente facilidad del sistema fónico del español se desmonta fácilmente en la práctica docente, donde las dificultades de producción residen más en la confrontación de los inventarios fonológicos de L1 y L2, que producen interferencias en la producción, que en las características fónicas propias de la lengua de enseñanza. En este sentido, diversos estudios dedicados a este fenómeno en lingüística contrastiva señalan la necesidad de tener en cuenta los fonemas de la L1 del estudiante para, a partir de sus características fonéticas, trabajar en las similitudes y diferencias en la L2 (Munro, 2018; Saito, Sun y Tierney, 2020).

Otras razones que intentarían explicar la poca atención dedicada a la pronunciación si la comparamos con otras destrezas comunicativas las encontramos en Cortés (2002), quien señala:

- La falta de conciencia sobre la importancia de la pronunciación por parte de los diseñadores de materiales y de los profesores que diseñan dichos contenidos.

- Una formación inadecuada del profesorado en el ámbito fónico y, sobre todo, en la didáctica de la pronunciación.

- Escasa atención a los fenómenos suprasegmentales y focalización en la articulación correcta de los fonemas.

- Creencia en los docentes de que la entonación es un fenómeno complejo, difícil de describir, o incluso imposible de enseñar a los aprendices de una lengua.

- Creencia de que la adquisición de la entonación se aprende de manera progresiva mediante la exposición a la L2.

En este sentido encontramos el trabajo de Usó (2009), quien señala el hecho de que un docente necesita saberse capacitado para poder enseñar de manera efectiva la L2. Es decir, que además de haberse formado en pronunciación, por ejemplo, y entender los procesos de articulación, entonación y acento, debe saber cómo explicarlos a sus estudiantes: necesita una formación en contenidos y en su didáctica. En definitiva, no basta solo con saber cómo hacer, sino de saber explicar para que otros sepan cómo poder hacerlo. Esta es la clave donde reside el trabajo de un buen docente, y en su simplicidad se esconde la dificultad real: para conocer un proceso aparentemente sencillo como es la pronunciación se deben comprender todos los mecanismos que se hayan implicados en ella, desde los anatómicos y fisiológicos hasta los pragmáticos y discursivos.

En relación con esta idea queremos señalar dos creencias erróneas bastante asentadas en el aprendizaje de una lengua extranjera: la primera, que para el correcto aprendizaje de la pronunciación de L2 basta con el modelo proporcionado por un hablante nativo; y la segunda, relacionada con la primera, que todos los hablantes de una lengua, por el 
hecho de saber hablarla, conocen cómo funciona esta. Pensemos en un hablante nativo de español sin conocimientos específicos sobre didáctica de la lengua y sin problemas de articulación a quien preguntemos, por ejemplo, cómo realiza el sonido $/ 1 /$. Seguramente la explicación que nos proporcione contendrá información sobre la posición aproximada de la lengua en la parte superior del paladar, pero si esa información no nos bastase para conseguir imitar el sonido, entonces seguramente se encontraría con que no tiene capacidad de poder explicar el sonido de otro modo y seguiría intentando que se le imitase y, al tiempo, tanto aprendiz como maestro cesarían en el intento. Apliquemos ahora este ejemplo a los docentes que son contratados para enseñar L2 solo por el hecho de ser nativos bajo la falacia perceptiva, asentada bajo la premisa de que la calidad del modelo proporcionado bastará, por sí solo, para que un hablante adquiera la competencia en pronunciación. Reiteramos la importancia de la formación en didáctica de la lengua no solo para conocer los contenidos, sino para saber cómo enseñarlos. No es posible enseñar un aspecto de la lengua si antes no se adquiere conciencia de cómo ha sido posible producirlo, con la necesaria reflexión metalingüística añadida. En el caso de la pronunciación, se debe tomar conciencia sobre los aspectos implicados en la articulación mecánica de los sonidos para poder comprender las posibles dificultades en su producción y, además, se han de tener en cuenta los matices enunciativos e interactivos asociados a los elementos suprasegmentales, tal y como han señalado Hidalgo y Cabedo (2012).

\section{La enseñanza de la pronunciación según los paradigmas lingüísticos}

Tradicionalmente, durante la hegemonía de los paradigmas generativistas y estructuralistas (hasta la irrupción de los modelos más vinculados con la lingüística cognitiva de finales de los años 80), en la enseñanza de una lengua se privilegió el aprendizaje de la gramática entendiendo que, de este modo, se dotaba a los estudiantes de elementos básicos con los que poder componer y comprender los enunciados. Se focalizaba la enseñanza de los aspectos morfológicos y sintácticos de la lengua con la idea de que los estudiantes estarían asentando sus conocimientos sobre la base del sistema en que se construía la lengua, en detrimento de los aspectos más dependientes del contexto: los elementos pragmáticos, las variedades diafásicas, los rasgos suprasegmentales o la correcta pronunciación de los sonidos de la lengua. De esta manera, en el abordaje de la enseñanza de una lengua el enfoque estructuralista diferenció los componentes gramaticales del lenguaje para, por un lado, poder focalizar y especializar el estudio de un módulo lingüístico mientras que, por otra, asumió que los estudiantes integrarían los conocimientos en el producto final: el dominio de una lengua. La herencia de los estudios estructuralistas en la perspectiva de enseñanzas lingüísticas se hace patente al comprobar la desigual dedicación en los manuales de enseñanza de español de los componentes identificados con la gramática y las situaciones de la lengua en uso o la pronunciación (Bartolí, 2015).

El modelo actual sigue un enfoque próximo al paradigma comunicativo funcional. Este enfoque privilegia que el estudiante de una lengua sea capaz de comunicar sus necesidades y asume que la corrección lingüística, ya sea desde el punto de vista gramatical o de la precisión en la selección y pronunciación léxica, es un hecho que devendrá de la práctica de la lengua en contextos funcionales. El énfasis en la capacidad de comunicación se focaliza en el significado, en la comprensión y expresión de 
mensajes comunicativos, por encima de la forma en todo el proceso de enseñanza (Cassany, 1999). Incluso la rectificación de errores en producción se realiza bajo la premisa de la distinción entre los errores de tipo comunicativo y los errores propiamente lingüísticos. Siguiendo a Llisterri (2011: sección de tipologías lingüísticas, párrafo 1), existiría una jerarquía de errores de producción que distinguiría entre:

1. Errores lingüísticos:

- Errores fonémicos: producidos por la diferencia de inventarios entre L1 y L2.

- Errores fonéticos: producidos por la diferente realización en cada lengua de fonemas comunes en L1y L2.

- Errores alofónicos: producidos por las diferentes variaciones de un fonema presentes únicamente en L1 o L2.

- Errores distribucionales: producidos por segmentos que existen en L1 y L2, pero con diferentes distribuciones.

2. Errores comunicativos:

- Errores que impiden la comunicación: la producción de un sonido actúa sobre un par mínimo y conlleva un cambio de significado. Por ejemplo, producir pelo en lugar de pero.

- Errores que dificultan la comunicación: la producción de un sonido no es del todo correcta, pero el receptor puede adivinar el fonema correcto teniendo en cuenta el contexto de producción. Por ejemplo, producir servesa en lugar de cerveza.

- Errores que no dificultan la comunicación: la producción de los fonemas, aunque no es la correcta, no conlleva dificultades de comprensión. Por ejemplo, producir aua en lugar de agua.

Los errores comunicativos serían los que se trabajarían de manera más activa al entender que podrían interferir en la ininteligibilidad del mensaje del estudiante de una lengua. En cambio, los errores lingüísticos se enmarcarían en el proceso natural de aprendizaje de una lengua y no constituirían, per se, un objeto de corrección lingüística. Sin embargo, como señala Padilla (2015), esta distinción entre errores conduce a una falacia fonética, pues no es posible establecer una separación entre el componente fonológico y el fonético. Es decir, en una situación de enseñanza de la pronunciación no podemos separar el componente considerado como más mecánico (articulación fonémica) de su esencia o representación fonológica, pues ambas van vinculadas y se manifiestan simultáneamente en la producción de cada elemento discursivo. Además, hay que tener en cuenta los valores pragmáticos adicionales que proporciona la diferente entonación de los fonemas, pues tal y como señalan Hidalgo y Cabedo (2012), el matiz entonativo se relaciona con los diferentes actos de habla en el plano interactivo del discurso. 


\section{La enseñanza de la pronunciación según el método utilizado}

Los métodos más consolidados para la enseñanza de la pronunciación se enmarcan en dos perspectivas que, si bien son complementarias, suelen aplicarse de forma diferenciada:

- La primera —métodos perceptivos - se basa en replicar los mecanismos naturales de adquisición del lenguaje y, por ello, su sistema de aprendizaje se basa en la percepción de los sonidos. Un ejemplo sería el método de oposiciones fonológicas.

- La segunda — métodos productivos - pone el foco de atención sobre la capacidad del hablante en producir el sonido, y se centra en la producción de los fonemas. Por ejemplo, el método fono-articulatorio.

Ambas perspectivas resultan necesarias para un aprendiz de lengua, por lo que defendemos la necesidad de integrar sus beneficios en una propuesta combinada que se describirá en el apartado 4. Antes, presentamos brevemente un estado de la cuestión con la descripción de las ventajas e inconvenientes que presenta la aplicación de los diferentes métodos según la bibliografía (Llisterri, 2011; Paredes García, 2013).

\subsection{Los métodos perceptivos}

Los métodos perceptivos se basan en los mecanismos de asimilación y disimilación de sonidos a través de la exposición auditiva a la L2 y su contraste con el inventario de L1. La psicología, la lingüística y la neurología cognitiva se han interesado por describir los procesos mediante los cuales un hablante es capaz de percibir, segmentar, identificar y dotar de significado a un sonido. Una visión que recoge estos postulados es la teoría del Modelo de Aprendizaje del habla en su versión revisada (Flege y Ocke-Schwen, 2021). Esta teoría busca explicar cómo los estudiantes de L2 adquieren y categorizan los sonidos de la lengua nueva una vez ha pasado el considerado como período crítico de adquisición del lenguaje, que en la versión inicial de Lenneberg (1967) se entendía como el período ideal para el aprendizaje lingüístico y se consideraba que los aprendizajes que se realizaran una vez finalizada la pubertad, y con la estructura neuronal ya consolidada, no serían lingüísticamente correctos (Hartshorne y Makovski, 2019).

En la actualidad se acepta la existencia de un período de facilitación del aprendizaje que se situaría entre los 2-12 años, pues tal y como señala Paredes Duarte (2020) en su revisión sobre las aportaciones de la neurolingüística al aprendizaje del lenguaje, los estudios de neuroimagen han aportado evidencias acerca de las diferencias de L2 cuando se ha aprendido en un entorno bilingüe en la niñez y cuando se ha aprendido después (en el entorno bilingüe, L1 y L2 comparten estructuras localizadas en el lóbulo frontal, mientras que en el caso de aprendizaje de L2 tardía las estructuras se localizan más hacia el lóbulo temporal). Así, según la teoría del modelo de aprendizaje del habla los estudiantes que adquieren una L2 posterior al aprendizaje de L1 clasifican los fonemas de L2 según el grado de familiaridad respecto a L1. Así, se distinguen tres agrupaciones de fonemas: fonemas que existen en ambos inventarios, fonemas similares a los existentes en L1, fonemas que no existen en L1. 
Según esta teoría, los estudiantes siempre intentarían reproducir los fonemas ya adquiridos y este sería el origen de sus problemas de pronunciación. Siguiendo este modelo, la enseñanza de la pronunciación debería partir de los fonemas nuevos para seguir hacia el aprendizaje de los sonidos similares, pues esta teoría incide en el hecho de que cuanto más parecidos sean los sonidos, mayor dificultad tendrá el estudiante para lograr diferenciar los correspondientes a L1 y L2. Estudios recientes han señalado que, si bien las lenguas que presentan sistemas fonéticos-fonológicos emparentadas se aprenden con mayor facilidad, en el caso concreto de la pronunciación los sonidos similares producen un efecto de interferencia que complican la adquisición lingüística (Lozano González, 2012; Castro Gavelán, 2018).

Otro método bien consolidado de enseñanza de la pronunciación es el de los pares mínimos, basado en los principios de la fonología estructural norteamericana. Se presenta una selección de pares de palabras con una estructura común, pero donde una de ellas presenta una variación en uno de los rasgos fonémicos que resulta en un cambio de significado, como por ejemplo /'pera/- /'pela/. El objetivo de este método es ayudar a que el estudiante identifique las variaciones mediante la semántica y se trabaja de forma aislada, sin integrar el componente suprasegmental (Llisterri, 2011).

La teoría clásica de la Sordera Fonológica (Polivanov, 1931) es otro método que ha sido muy utilizado para abordar las cuestiones de la enseñanza de la pronunciación en L2. Esta teoría perceptiva parte del campo de los trastornos del lenguaje y asimila la situación de un estudiante de L2 a la de una persona con deficiencias auditivas, pues en ambas situaciones la persona percibiría de manera incompleta las propiedades de los sonidos, siendo incapaz por tanto de reproducir con éxito el modelo. Es decir, el aprendizaje de la pronunciación debería de basarse en la exposición y discriminación auditiva de los sonidos antes que en su articulación. Destacamos esta teoría por las ayudas técnicas que se desarrollaron para mejorar la percepción y la discriminación auditiva, como el SUVAG ${ }^{1}$ (Sistema Universal Verbal Audiotonal de Guberina). Es un aparato que se coloca en los oídos del estudiante con unos auriculares y filtra la intensidad adecuada para cada sonido, con el objetivo de facilitar la percepción del sonido. En el caso de la propuesta que se realiza en el apartado 4, recomendamos la utilización de una variación de esta técnica que combina la información perceptiva con la información vibrotáctil mediante la incorporación de una pulsera vibradora en la muñeca del estudiante que transforma el sonido que se recoge por el micrófono en vibraciones, permitiendo así que el estudiante pueda controlar la intensidad, el ritmo, el acento o la duración del sonido. Este sistema se utiliza de forma habitual en las intervenciones clínicas de problemas perceptivos, por lo que su aplicación al ámbito de ELE puede resultar muy beneficiosa para la mejora de la discriminación auditiva.

\footnotetext{
${ }^{1}$ El método SUVAG se compone por unos auriculares y un micrófono más un programa que se puede instalar en cualquier dispositivo electrónico. A través de los auriculares, el estudiante percibe de forma nítida y sin ruidos externos el sonido producido a través del micrófono. El programa dispone de una serie de filtros electrónicos que permiten modificar la recepción del sonido según la frecuencia.
} 


\subsection{El método productivo o fonoarticulatorio}

El método productivo se basa en la premisa de que es necesario conocer bien el sistema fonador y las necesidades articulatorias de un sonido antes de poder producirlo de manera correcta. Este método es el que predomina en el área de la rehabilitación logopédica, donde la enseñanza de los sonidos se apoya en la fonética articulatoria. Es un método eficaz, pero lento y requiere que el docente esté formado en principios de fonética y fonología al tiempo que en didáctica de la enseñanza de la producción oral. Dos han sido las principales objeciones que se han señalado en el método fonoarticulatorio (Llisterri, 2011: problemas de los métodos articulatorios):

- La exigencia de una especialización y una dedicación consciente por parte del estudiante para que, conociendo el funcionamiento de su sistema fonador, pueda practicar el aprendizaje articulatorio necesario para la pronunciación de la lengua. Este aprendizaje requiere un entrenamiento articulatorio selectivo, por lo que algunos estudios lo consideran como un método poco motivacional o lúdico. Sin embargo, es el método más utilizado en Logopedia para la rehabilitación de los problemas de pronunciación (dislalias) $\mathrm{y}$, sin duda, una de las claves para mantener el componente motivacional en los estudiantes es una adecuada formación en didáctica de la enseñanza de la lengua, pues la enseñanza de rutinas o movimientos articulatorios se refuerza con movimientos propioceptivos que ayudan a la interiorización de los patrones articulatorios.

- El enfoque centrado en la articulación fonemática, pues al trabajar con la articulación de palabras aisladas se ignoran los componentes suprasegmentales como el acento o la prosodia.

Este método exige, efectivamente, un aprendizaje consciente por parte del estudiante que debe basarse en su propiocepción de la pronunciación.

\section{Propuesta de enseñanza de la pronunciación a través de la propiocepción}

La propuesta didáctica de enseñanza de la pronunciación que presentamos es una propuesta heterogénea cuya base es la consideración de que la articulación se constituye por un acto motor imposible de realizar sin la conciencia de las propiedades perceptivas del sonido. Es decir, para poder enseñar un sonido es necesario atender a la vez al componente perceptivo y al expresivo: consideramos que una propuesta de enseñanza que solo se dirija a uno de los dos componentes constituirá una enseñanza incompleta del proceso de pronunciación. Por este motivo, partimos de un aprendizaje motor de la articulación para asentar la base productiva del sonido y basamos nuestra propuesta de intervención en la propiocepción, entendida como un proceso complejo por el cual nuestro cuerpo toma conciencia de la situación y los movimientos de los músculos implicados en la realización de una acción.

La propuesta perceptivo-expresiva que presentamos se basa en los principios de la psicolingüística cognitiva; concretamente, en el modelo de doble ruta de procesamiento del lenguaje escrito (Marshall y Newcombe, 1973; Coltheart, 1980; Cuetos, 1998; Hickok y Poeppel, 2004). Este modelo trata de explicar los procesos cognitivos de base perceptiva que se producen en el acto lector asumiendo que el producto lingüístico no es sino la suma de diversos procesos independientes, pero relacionados entre sí. Este modelo explicativo ha resultado muy productivo en el área de la evaluación e 
intervención en la reeducación de la lectura, pues la concepción modular permite la identificación del proceso secuencial donde el hablante presenta problemas para poder intervenir sobre los elementos alterados $\mathrm{y}$, de ese modo, proceder a la rehabilitación lingüística (Cuetos, 2011).

En nuestra adaptación del modelo de doble ruta pasamos de un modelo que explica el procesamiento cognitivo de la lectura al procesamiento cognitivo de la pronunciación. Además, añadimos la importancia de la producción oral, pues el modelo de lectura tan solo consideraba, en sus inicios, el procesamiento de input lingüístico, pero sin considerar el procesamiento del output lingüístico. Existen trabajos que han utilizado el modelo de la doble ruta para explicar el procesamiento oral y el escrito. Summer at al. (2014), por ejemplo, aplicaron la hipótesis de la doble ruta para explicar la variabilidad fonética en tareas orales de repetición y percepción/producción. Sin embargo, en este trabajo perseguimos una finalidad aplicada: la posibilidad de aplicar el modelo de doble ruta a la enseñanza de pronunciación en L2.

Presentamos un modelo que desgrana las secuencias cognitivo-perceptivas que sigue un hablante al procesar el habla al tiempo que consideramos los requisitos anatómicofisiológicos y la propiocepción activa que precisa un hablante para poder producir un sonido determinado de una lengua. Mediante este modelo descriptivo se facilita la identificación de los elementos que resultan más problemáticos para un hablante para, de este modo, poder entrenar específicamente esos elementos mediante las directrices de la didáctica de la enseñanza de la lengua en pronunciación.

\subsection{Procesamiento cognitivo del sistema perceptivo de la producción}

Al percibir una secuencia de sonidos en un contexto discursivo o en una palabra aislada, el primer paso del procesamiento es la integración auditiva correcta de la cadena fónica. Poder integrar los sonidos supone tener la capacidad de discriminar los rasgos pertinentes que componen cada fonema, además de identificar la intensidad, la duración, el acento y la prosodia aplicada por el emisor a los sonidos percibidos.

Si el hablante es capaz de percibir, discriminar e identificar correctamente todos los rasgos perceptivos, entonces podrá reconocer la estructura fónica de la secuencia y procesará la secuencia mediante la ruta global. Esta ruta le permite acceder de forma directa a su almacén de léxico auditivo, donde se encuentran almacenadas las palabras que han sido percibidas a lo largo de nuestro aprendizaje. Estas secuencias fónicas serán más fáciles de activar en función de la frecuencia, la familiaridad de aparición y la edad a la que se haya adquirido la palabra (Bargetto y Riffo, 2019; Santos, Trigo y Romero, 2020). Si la secuencia de sonidos se identifica como un conjunto previamente almacenado, entonces la ruta global permitirá asociar de manera directa el soporte fónico con su correlato semántico. Por el contrario, si un hablante presenta problemas relacionados con la integración auditiva o no es capaz de reconocer los sonidos que integran una secuencia, entonces no podrá utilizar la ruta global, más directa, y deberá utilizar la ruta fonológica. En esta ruta se analizarán de manera independiente los rasgos que el hablante haya percibido para poder asociarlos con un sonido. Si la asociación es exitosa y el hablante es capaz de integrar los sonidos y reconocerlos como unidad auditiva, entonces podría acceder a la ruta global. En cambio, si solo consigue identificar algunos de los sonidos el siguiente paso sería realizar la conversión de los 
fonemas a grafemas para, de este modo, acudir al léxico visual, una especie de almacén donde se hayan registradas las palabras escritas que hemos asimilado a lo largo de nuestro aprendizaje. Una vez ahí, se debería poder reconocer la forma de la palabra, lo que permitiría acudir al sistema semántico.

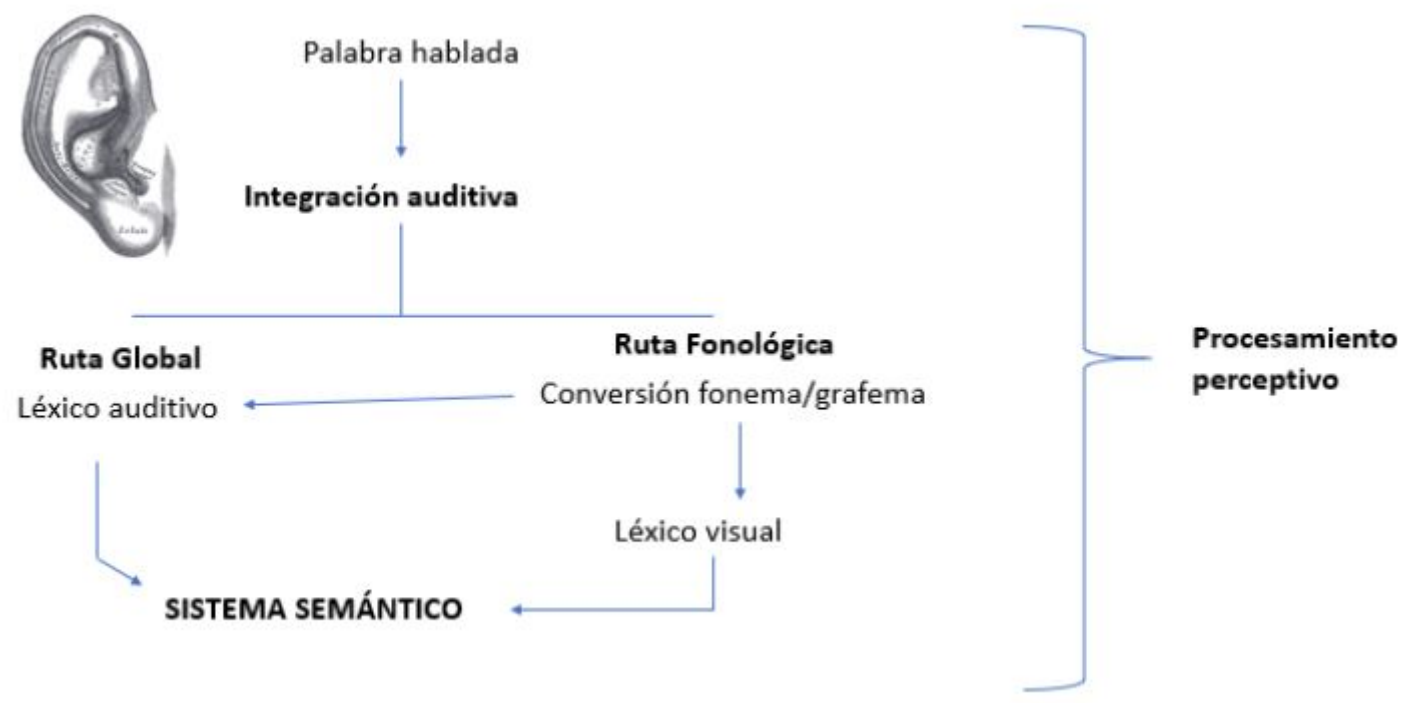

Figura 1. Representación gráfica del modelo de doble ruta del procesamiento perceptivo Fuente: autoría propia a partir del modelo de doble ruta del procesamiento de la lectura (Cuetos, 2008)

En el párrafo anterior hemos descrito las opciones de procesamiento perceptivo de la pronunciación. Pero, por supuesto, en cada una de las secuencias señaladas un estudiante puede presentar problemas para el procesamiento perceptivo, y deberemos tener en cuenta sus particularidades para poder diseñar una propuesta de intervención adecuada:

- Problemas relacionados con la integración auditiva: en el caso de que un hablante presente problemas para la discriminación de uno o varios sonidos, se recomienda el uso del dispositivo SUVAG con complementación vibrotáctil y un entrenamiento específico con actividades basadas en pares mínimos para ayudar en la identificación correcta de los sonidos.

Se recomienda que la intervención en propiocepción perceptiva se complemente con una intervención que refuerce la producción articulatoria de los sonidos problemáticos, ya que de este modo se facilitará la integración de la información en las dos vertientes. La intervención en producción primero se centrará en la articulación correcta del sonido producido de manera aislada, pero después deberá integrar ese sonido en sílabas directas, inversas y trabadas para poder practicar su producción en palabras y oraciones. De este modo, el estudiante se podrá familiarizar con las variaciones perceptivas y articulatorias que se producen en los sonidos en función de los fonemas que lo acompañen.

- Problemas relacionados con el léxico auditivo: en el caso de que en un contexto léxico determinado el hablante no percibiera alguno de los sonidos, pero sí fuera capaz de integrarlo en un conjunto léxico, entonces la propiedad de 
cierre gestáltica le permitiría recuperar palabras similares a la percibida. Este sería el caso de hablantes con un buen dominio léxico-semántico de la lengua, quienes, aunque no dominen todos los sonidos de una lengua son capaces de identificar las secuencias léxicas y, por tanto, de acceder al significado del discurso hablado. Por el contrario, podemos tener el caso de hablantes que siendo capaces de identificar los sonidos que componen la secuencia fónica, no son capaces de integrar los sonidos en las palabras. En este caso, estaríamos ante un caso donde se debería priorizar el conocimiento léxico y completar la intervención con un apoyo gráfico de la palabra escrita para reforzar al mismo tiempo el léxico visual, sin olvidarnos de reforzar el acento, el tono y la prosodia adecuadas.

Las actividades de refuerzo adecuadas para los problemas en el acceso al léxico auditivo serían aquellas que permitieran unir las secuencias fónicas con su significado. Por tanto, cabe ampliar el vocabulario para facilitar el acceso léxico. La actividad recomendada sería trabajar en la ampliación de vocabulario con palabras seleccionadas bajo un criterio de familiaridad, centro de interés del estudiante y que contengan los sonidos que no se dominan. Se recomienda la presentación de la secuencia tanto en su forma fónica como gráfica para fomentar que el almacenamiento de la información se realice bien por vía visual, bien por vía auditiva.

- Problemas relacionados con el mecanismo de conversión fonema/grafema: nos encontramos ante este problema cuando un hablante se enfrenta a un fonema nuevo o diferente en L2 respecto a su inventario de L1. Como señalan los estudios ya comentados en el apartado anterior, los hablantes tenderán a intentar asimilar los rasgos de los fonemas nuevos a fonemas preexistentes en su L1. Pensamos que se debe aprovechar esta estrategia en la enseñanza de los fonemas mediante actividades que refuercen el contraste entre esos sonidos para que, de este modo, el hablante pueda diferenciar mejor entre los sonidos parecidos. Recomendamos el uso de dispositivos que faciliten la percepción de los rasgos fonemáticos como el SUVAG junto con actividades de pares mínimos y de producción articulatoria tanto en contextos léxicos aislados como discursivos que se apoyen en la representación gráfica de los fonemas. De este modo, se incide en el aprendizaje de los sonidos al tiempo que se establece su relación con el grafema, mejorando así la representación en el léxico visual y en el léxico auditivo.

- Problemas relacionados con el léxico visual: nos encontramos ante estas dificultades cuando un hablante es capaz de identificar de manera correcta los sonidos de una secuencia, pero no de reconocer el significado asociado a ella. La estrategia que sigue entonces el hablante es la de activar el mecanismo de conversión de los fonemas en grafemas y acudir al léxico visual, donde el hablante puede llegar a identificar el significado de la secuencia mediante los grafemas. En este nivel, los problemas se producen con las palabras poco familiares compuestas con grafemas arbitrarios: el hablante identifica los fonemas, pero solo es capaz de acceder al significado a través de la representación gráfica de la palabra. 
Las actividades deberían centrarse en reforzar la asociación de los grafemas con los fonemas, incidiendo en las situaciones de relación arbitraria. Se debe fomentar la amplitud de vocabulario partiendo de selecciones de palabras próximas al centro de interés del hablante que contengan los sonidos problemáticos representadas de forma gráfica. Aunque el acceso a la semántica se realice por la vía gráfica, también se debe incidir en la vía auditiva para reforzar el afianzamiento de la conversión grafema-fonema y el aprendizaje mediante el léxico auditivo tanto en entornos léxicos como discursivos.

\subsection{Procesamiento cognitivo del sistema productivo del lenguaje hablado}

Cuando queremos producir un discurso, lo primero que hacemos es recurrir al almacén perceptivo, donde se encuentra almacenada toda la información segmental $y$ suprasegmental de los sonidos. Al acudir al almacén del sistema perceptivo, identificamos y seleccionamos los elementos adecuados al discurso que queremos producir en función de la ilocutividad comunicativa. Para ello, es necesario que el hablante previamente haya aprendido e integrado los rasgos pertinentes de los fonemas de la lengua. En el caso de que un hablante conozca los sonidos que necesita producir para transmitir de manera adecuada su mensaje, realizará la selección de acuerdo con un criterio semántico-pragmático y tendrá almacenada en el lexicón auditivo la información que precisa para realizar una pronunciación correcta. La pronunciación de un sonido no se limita solo a las cuestiones articulatorias, sino que comprende las variaciones articulatorias de acuerdo con la combinación fonemática, el acento, la intensidad, el tono, la duración y la prosodia adecuadas a la intención comunicativa. Puede suceder, no obstante, que un hablante conozca la mayoría de los sonidos de una lengua y que acuda al sistema semántico para seleccionar las palabras que compondrán su discurso, pero no logre producir las características de pronunciación correctas. Según los estudios ya comentados, el hablante intentará entonces producir el sonido más aproximado al sonido ideal de los disponibles en su registro perceptivo y teniendo en cuenta sus posibilidades articulatorias para, de este modo, intentar que la pronunciación resultante pueda ser reconocida por el emisor mediante el contraste con las secuencias almacenadas en su léxico auditivo. En tal caso, el hablante produciría errores comunicativos que no interferirían en el proceso de comunicación. Desde el enfoque comunicativo se considera que este tipo de problemas son consustanciales al proceso de aprendizaje de una lengua; sin embargo, en nuestra propuesta sí consideramos que es importante incluirlos en un programa de mejora de la pronunciación puesto que debemos tener en cuenta el hecho de que cuando un hablante no domina la enunciación de su discurso, entonces esta descansa sobre la voluntad de cooperación comunicativa del emisor. Cuanto mejor sea la pronunciación del emisor, mayores serán las probabilidades de que la ilocutividad discursiva llegue al interlocutor sin necesidad de realizar inferencias o interpretaciones del mensaje.

La pronunciación es uno de los elementos más importantes desde el punto de vista comunicativo de la lengua, pues resulta determinante para la inteligibilidad del discurso. Por este motivo, es necesario incidir en su enseñanza en el proceso de aprendizaje de la lengua oral desde un enfoque funcional y discursivo que focalice el entrenamiento mecánico de la articulación en un contexto discursivo próximo al estudiante (GonzálezGarcía, 2019). En el marco del aprendizaje más mecánico, como es el entrenamiento de 
las habilidades articulatorias, el reto estriba en enseñar al estudiante a reconocer y saber controlar de manera consciente los movimientos anatómicos y fisiológicos necesarios para la producción de los sonidos. La propiocepción es un elemento clave a la hora de hacer comprender al estudiante la secuencia exacta de movimientos y fuerza que precisa la articulación de cada sonido de manera aislada o combinada. Algunos estudios (vid. Estado de la cuestión en Twomey y Angelosi (2020) han comprobado la importancia de la conciencia basada en la propiocepción en el aprendizaje de los fonemas de L1 incluso en el marco de la lingüística computacional. En el citado estudio, se demuestra que los robots aprenden más rápido teniendo la referencia de su propio cuerpo en comparación con modelos computacionales incorpóreos.

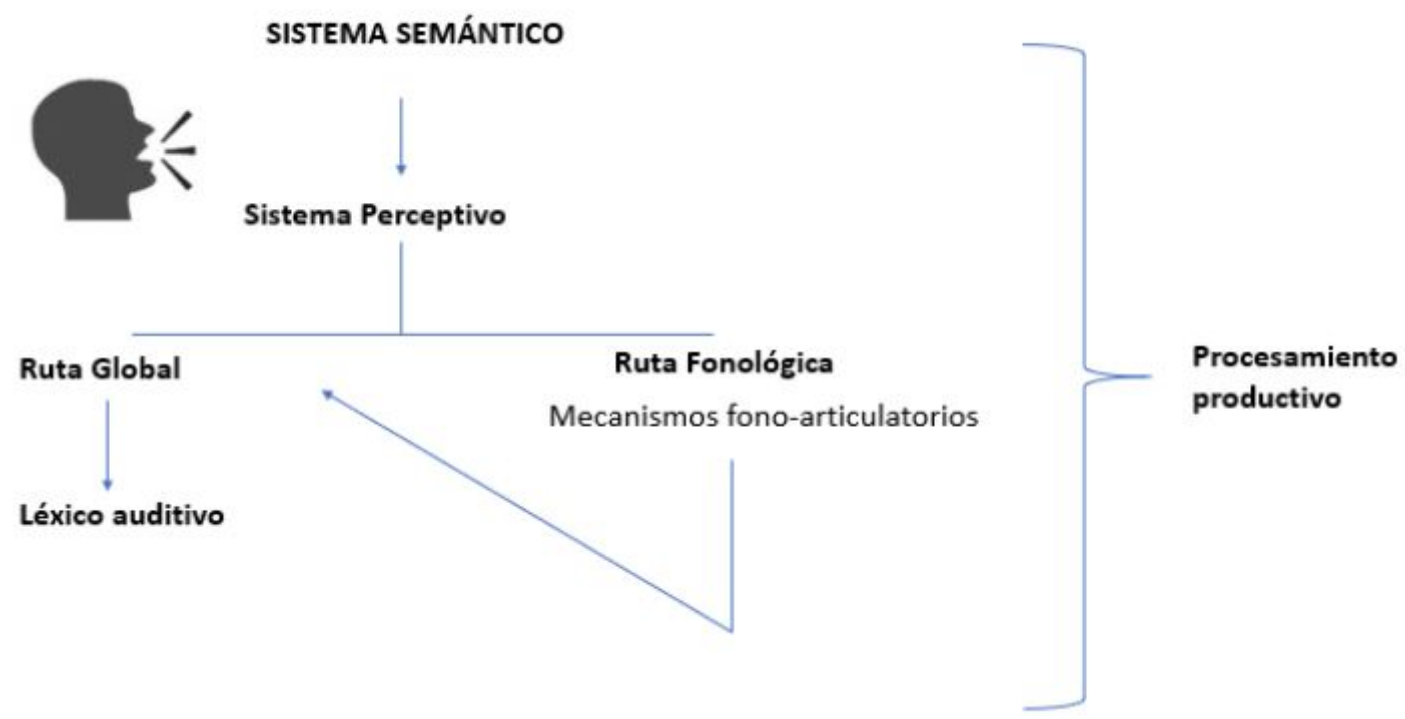

Figura 2. Representación gráfica del modelo de doble ruta del procesamiento productivo Fuente: autoría propia a partir del modelo de doble ruta del procesamiento de la lectura (Cuetos, 2008)

A continuación, se desarrollan algunos de los problemas que se pueden presentar en el proceso de aprendizaje de la pronunciación de una lengua y las acciones que se proponen para paliar estas dificultades:

- Problemas relacionados con el sistema perceptivo: si un hablante desconoce los sonidos que debe pronunciar, entonces deberá buscar fórmulas alternativas para comunicarse como la expresión mediante parafasias o circunloquios. Si lo que no domina son algunos de los rasgos vinculados a un fonema, entonces la estrategia que seguirá es buscar en el almacén de su sistema perceptivo los sonidos que más se asimilen al sonido ideal según sus posibilidades articulatorias. Esta selección puede resultar adecuada o no, dependiendo de la relación de semejanzas fonémicas que exista entre L1 y L2.

Las actividades recomendadas para este nivel serían las centradas en la propiocepción de los rasgos distintivos de cada fonema. En primer lugar, se debería trabajar la adecuada percepción fonemática, como ya se ha explicado en el apartado anterior. Al mismo tiempo que se trabaja sobre los mecanismos perceptivos, se debe incidir en la conciencia del hablante sobre los movimientos articulatorios que realiza para pronunciar determinado sonido. Estos ejercicios se 
explican con más detalle en el apartado dedicado a los problemas fonoarticulatorios.

- Problemas relacionados con el léxico auditivo: un hablante presentará problemas en esta área cuando, a pesar de identificar los rasgos de los sonidos, no sea capaz de integrarlos en los fonemas correspondientes y, por tanto, no pueda relacionarlos con las secuencias fonémicas enunciadas. Es decir, cuando a pesar de reconocer todos o la mayoría de los sonidos, no se reconozcan las unidades léxicas que componen. En este caso, se debería ampliar la exposición a secuencias de percepción auditiva (ejercicios de recepción auditiva en entornos conversacionales) al tiempo que se relaciona la forma auditiva con la secuenciación fonemática. Se recomienda comenzar por unidades léxicas y, una vez el estudiante esté familiarizado con estas unidades, pasar al nivel discursivo para reforzar el aprendizaje de los rasgos suprasegmentales. Así, si el estudiante es capaz de integrar la información segmental y suprasegmental cuando tenga que acceder al léxico auditivo para recuperar la secuencia almacenada, dispondrá de toda la información adecuada para poder realizar una pronunciación adecuada a su ilocutividad.

- Problemas relacionados con los mecanismos fonoarticulatorios: Esta sería la parte más teórica y mecánica de la intervención, y debería componer una parte sustancial de los ejercicios para la mejora de la pronunciación en las personas que están adquiriendo una lengua. La base de la intervención se fundamenta en la auto-conciencia del estudiante sobre la propiocepción cinestética. En primer lugar, se explica de manera visual qué movimientos y qué fuerza se precisa para poder reproducir el punto, modo y sonoridad del fonema deseado y, en segundo lugar, se contrasta la realización ideal con la pronunciación de la que se parte. Este aprendizaje propioceptivo se debe realizar junto a ejercicios anatómicos en el caso de que se precise (por ejemplo, ejercicios para reforzar la fuerza del ápice lingual) y ayudándose de la utilización de gestos visuales de apoyo a la articulación, tal y como se han venido utilizando en el método verbo-tonal. Estos gestos se utilizan de manera habitual en el área de la rehabilitación lingüística y se utilizan para apoyar la enseñanza y la producción de los sonidos. Existen diferentes tipologías basadas en los preceptos de la comunicación bimodal, pero recomendamos los inventarios de Monfort, Rojo y Juárez (1982) y de Vides Vargas (2013) por su versatilidad y facilidad de aprendizaje. Estos gestos presentan una doble función: por un lado, destacan un rasgo fonatorio principal que posibilita la realización del sonido y, por otro, dotan de corporeidad a un aprendizaje abstracto, facilitando así su aprendizaje.

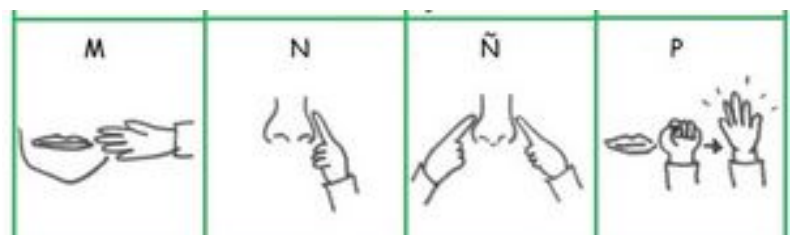

Figura 3. Gestos de apoyo a la pronunciación Fuente: Monfort, Rojo y Juárez (1982) 


\section{Programa de intervención propioceptivo}

Se presenta un programa de intervención basado en el modelo de doble ruta de procesamiento de la pronunciación presentado en los apartados anteriores. El objetivo del programa es ofrecer un plan personalizado de enseñanza que pueda reforzar las áreas tanto perceptivas como productivas que resulten complicadas para el estudiante de L2. Se propone una práctica diseñada para sesiones individuales centradas en las dificultades de pronunciación de cada estudiante.

En primer lugar, es necesario realizar una evaluación individualizada de las necesidades de cada estudiante, independientemente del nivel de conocimiento de lengua que presenten. Por ello, se recomienda que en la primera sesión se expliquen los objetivos que se persigue con la evaluación para así hacer consciente al estudiante de la necesidad de su implicación activa en el aprendizaje de la pronunciación mediante métodos propioceptivos. Tras esta explicación, se realiza una evaluación de la producción con el registro fonológico inducido (Juárez y Monfort, 2001) seguida de una actividad de producción espontánea del lenguaje oral del estudiante en un contexto conversacional interactivo con el evaluador para que éste para comprobar cuáles son los sonidos más problemáticos para cada estudiante en el nivel oracional y la producción léxica. Como método complementario se recomienda preguntar directamente a los estudiantes qué sonidos les resultan más complicados para poder contrastar la información subjetiva propioceptiva con los registros objetivos de la evaluación.

Tras la evaluación, el docente deberá analizar los resultados y proponer un plan de perfeccionamiento de la pronunciación personalizado para el estudiante. La propuesta que defendemos tendría una duración ideal de 10 sesiones presenciales de 25 minutos que se complementarían con el envío por parte del estudiante de dos vídeos semanales de un máximo de 1 minuto de duración donde se mostrara la práctica en un contexto discursivo de los sonidos trabajados en el aula. Estos vídeos tendrían una doble función: por un lado, monitorizar que el estudiante practica de manera correcta las tareas propuestas y, en segundo lugar, para que el docente pueda proporcionar retroalimentación y, en caso necesario, reorientar al estudiante.

Las 2 primeras sesiones de enseñanza de la pronunciación se deben centrar en que el estudiante adquiera la propiocepción de los movimientos que realiza con su aparato fonador y los contraste con los movimientos requeridos en la producción correcta de los sonidos donde se presentan dificultades. Así, la primera sesión sería la más teórica y se destinaría a explicar la anatomía y fisiología del aparato fonador con el objetivo de que el estudiante adquiera las nociones básicas donde asentar su aprendizaje propioceptivo sensoriomotriz. Así, se demandaría al estudiante que describiera los movimientos que realiza con su aparato fonador al intentar articular el sonido deseado y, a continuación, se realizarían actividades de contraste con la realización aproximada pronunciada de manera espontánea. Tras esta explicación teórica, se recomienda la práctica productiva del contraste fonemático con el SUVAG con complemento vibrotáctil, pues de esta manera el estudiante percibirá de manera sensorial las diferencias que se relacionan con la pronunciación diferenciada de los fonemas tanto en el nivel segmental como en el suprasegmental. Si se comprobara que el estudiante manifiesta algún problema de tipo anatómico o fisiológico para poder realizar de manera adecuada un sonido, entonces se deberían realizar actividades centradas en este problema para ayudar a conseguir la pronunciación adecuada. Por ejemplo, se puede dar el caso de que los músculos 
bucofonatorios presenten hipotonía (bajo tono muscular) y se complique la producción de fonemas labiales oclusivos. En este caso, convendría trabajar de manera previa en la consecución de una tonificación muscular que permita realizar la pronunciación de los sonidos.

Las sesiones 3-10 seguirían el esquema que se describe a continuación: en los primeros 5 minutos se preguntaría al estudiante sobre las cuestiones que han resultado más complejas para él de las sesiones anteriores, y sobre los ejercicios que debía practicar en casa. De esta manera, se pueden orientar las actividades hacia las dificultades subjetivas manifestadas por el estudiante. Los siguientes 5 minutos se dedicarían a la práctica de los sonidos problemáticos de manera aislada hasta conseguir la correcta articulación en punto, modo y sonoridad con la ayuda de las directrices del docente y los gestos visuales de apoyo a la pronunciación. Para reforzar la propiocepción receptiva correcta del sonido, se utilizarán las ayudas de un amplificador de sonido SUVAG complementado por un dispositivo de vibración sujetado en la muñeca del estudiante. De este modo el estudiante puede percibir con mayor nitidez las variaciones en la producción de los sonidos tanto a través del sistema auditivo como a través del sistema táctil y realizar un ejercicio de retroalimentación en cuanto al tono, duración, acento e intensidad adecuadas de la pronunciación.

Una vez conseguida la aproximación articulatoria al sonido aislado, entonces se integraría en una secuencia de consonante seguida por las vocales en estructura de sílaba directa (3 minutos). En los siguientes 7 minutos se practicaría la adecuada producción de los sonidos en un entorno de palabra, siguiendo un criterio distribucional en actividades de pares mínimos: se trabaja la articulación del sonido en una selección de 5-15 palabras donde el sonido se situase en posición inicial, media o final de palabra. En esta fase, se recomienda que el sonido se encuentre en una secuencia de sílaba simple directa o inversa, nunca en sílabas trabadas.

Los últimos 5 minutos se dejarían para integrar la práctica articulatoria de las palabras seleccionadas en secuencias discursivas oracionales con pares mínimos. Por ejemplo, si trabajamos la diferenciación de «alto» frente a «harto», se propondría una práctica de la pronunciación en una oración como «estoy harto de ser tan alto» en el aula y después se pedía al estudiante que, a lo largo de la semana, enviara dos vídeos donde aparecieran palabras que necesariamente debían contener los sonidos trabajados en las sesiones previas.

Tras la realización de las 10 sesiones de intervención para la mejora de la pronunciación, se volvería a evaluar la pronunciación del estudiante mediante el registro fonológico inducido y la interacción oral espontánea. El objetivo de la re-evaluación no es otro que el de ajustar los objetivos de la intervención a las necesidades en pronunciación del estudiante. La propuesta de intervención en la pronunciación que presentamos consiste en un total de 12 sesiones de 25 minutos: una sesión de evaluación, diez de intervención y otra última de re-evaluación.

\section{Consideraciones finales}

Este trabajo presenta la justificación teórica de la propuesta para la mejora en pronunciación de español como L2. Es un modelo holístico que integra diversas aportaciones de los campos de la psicolingüística, la lingüística y la didáctica de la 
lengua. Además, la propuesta proporciona un modelo que interviene de forma conjunta sobre los procesos perceptivos y productivos de la pronunciación. La principal limitación de este trabajo es que, por el momento, es una propuesta teórica que debe ser aplicada a la enseñanza de la pronunciación en un contexto real de aula. Futuras investigaciones nos permitirán ahondar en los beneficios de esta propuesta, así como constatar las cuestiones mejorables.

\section{Bibliografía}

BARgetto Fernández, Miguel Ángel y RifFo OCAREs, Bernardo. 2019. «El reconocimiento de palabras y el acceso léxico: revisión de modelos y pruebas experimentales». Boletín de filología, 54(1), 341-361. https://dx.doi.org/10.4067/S071893032019000100341

BARTOLÍ, Marta. 2015. «Las actividades de pronunciación en los manuales de ELE». CAUCE. Revista Internacional de Filología, Comunicación y sus Didácticas, 38, 17-34.

CASSANY, Daniel. 1999. «Los enfoques comunicativos: enfoque y crítica». Lingüística y Literatura, 36-37, 11-33.

CASTRO GAVElÁN, Luis Ángel. 2018. La enseñanza-aprendizaje del español a angloamericanos mediante un enfoque comunicativo interactivo. Tesis doctoral. Madrid: Universidad Complutense de Madrid.

Coltheart, Max. 1980. Deep dyslexia: a review of the syndrome. En M. Colheart, K. Patterson y J. Marshall (eds.): Deep Dyslexia. Londres: Routledge y Kegan Paul, 58-74.

CORTÉS MoReno, Max. 2002. Didáctica de la prosodia del español: la acentuación y la entonación. Madrid: Edinumen

Cuetos, Fernando. 2008. Psicología de la lectura. Madrid: Praxis.

Cuetos, Fernando. 2011. Neurociencia del lenguaje: bases neurológicas e implicaciones clínicas. Madrid: Editorial Médica Panamericana.

GIL FERNÁNDEZ, Juana. (2007). Fonética para profesores de español: De la teoría a la práctica. Madrid: Arco/Libros.

GONZALEZ GARCÍA, Virginia. 2019. «Enseñar la producción oral». En L. Ruiz de Zarobe y Y. Ruiz de Zarobe (eds.) Enseñar hoy una lengua extranjera. Barcelona: Octaedro, 254-293

GOSPODARIC, Katarina. 2007. «La enseñanza de la pronunciación del español como lengua extranjera para eslovenohablantes». En E. Balmaseda (ed.), Las destrezas orales en la enseñanza del español L2-LE. XVII Congreso Internacional de la Asociación para la Enseñanza del Español como Lengua Extranjera (ASELE). Logroño: Servicio de Publicaciones de la Universidad de La Rioja, 583-596.

Flege, James y OcKe-Schwen, Bohn. 2021. «The Revised Speech Learning Model (SLM-r)». Pre-print. Recuperado el 29/07/2021, de https://www.researchgate.net/publication/348265785

LENNEBERG, Eric. 1967. Fundamentos biológicos del lenguaje. Madrid: Alianza Editorial. 
LLISTERRI, Joaquim. 2003. «La enseñanza de la pronunciación». Cervantes. Revista del Instituto Cervantes en Italia, 4(1), 91-114. Recuperado el 31/08/2021, de http://liceu.uab.cat/ joaquim/publicacions/Llisterri_03_Pronunciacion_ELE.pdf

LLISTERRI, Joaquim. 2011. «El análisis de los errores de producción». Recuperado el 27/07/2021, de

http://liceu.uab.cat/ joaquim/applied_linguistics/L2_phonetics/Analisis_Errores.html

LOZANO GONZÁlEZ, Lidia. 2012. Adquisición de terceras lenguas y lenguas adicionales. El proceso de comprensión escrita. Tesis doctoral. Barcelona: Universidad Autónoma de Barcelona

HARTSHORNE Joshua, MAKOVSKI Tal. 2019. "The effect of working memory maintenance on long-term memory». Mem Cognit, 47, 4, 749-763. doi: $10.3758 / \mathrm{s} 13421-019-00908-6$

HidAlgo NAVARro, Antonio. 2015. «Enseñar la entonación en E/LE: problemas, desafíos y propuesta de soluciones». Foro de profesores de E/LE, 11, 171-188.

Hidalgo Navarro, Antonio y CABedo, Adrián. 2012. La enseñanza de la entonación en el aula de E/LE. Madrid: Arco/Libros.

Juárez, Adoración y Monfort, Marc. 2001. Registro fonológico inducido. Madrid: Editorial CEPE.

MARSHALL, John y NeWCOMBE, Freda. 1973. «Patterns of paralexia: A psycholinguistic approach». Journal of Psycholinguistic Research, 2, 175-200.

Monfort, Marc.; Rojo, Ángel. y JuÁRez, Adoración. 1982. Programa elemental de comunicación bimodal para padres y educadores. Madrid: CEPE editorial.

Munro, Murray. 2018. «How Well Can We Predict Second Language Learners' Pronunciation Difficulties?» CATESOL Journal, 30, 1, 267-281.

OTERo Doval, Herminda. 2013. «Plurilingüismo y enseñanza de ELE en contextos multiculturales». En S. Borrell, B. Flecua, B. Crous y F. Sierra (Eds.), XXIII Congreso Internacional ASELE. Madrid: ASELE, 664-675.

PADILla GARCÍA, Xosé. 2015. La pronunciación del español: fonética y enseñanza de lenguas. Alicante: Servei de publicacions de la Universitat d'Alacant.

PAREDES GARCÍA, Florentino. 2013. «La enseñanza de la pronunciación en Español como Lengua Extranjera: cuestiones de método», Lingüística en la red, 11. Recuperado el 27/07/2021, de http://www.linred.es/monograficos_pdf/LR_monografico11-2/articulo3.pdf

PAREDES DuARTE, María Jesús. 2020. «El aprendizaje de lenguas desde una perspectiva neurolingüística». Sabir. International Bulletin of Applied Linguistics, 1, 2, 177-197.

Poch Olivé, Dolors. 2009. «La corrección fonética en español como lengua extranjera», marcoELE, 8, 193-200.

PolIVANOV, Evgueni. 1931. «La perception des sons d'une langue étrangère«. Travaux du Cercle Linguistique de Prague, 4: Réunion phonologique internationale tenue à Prague (18-21/ XII 1930), 4, 79-96. 
SAITO, Kazuya; SuN, Hui y TIERNEY, Adam. 2020. «Domain-general auditory processing determines success in second language pronunciation learning in adulthood: A longitudinal study». Applied Psycholinguistics, 41, 5, 1083-1112

SANTAMARÍA BUSTO, Enrique. 2010. «Formación y recursos para la enseñanza de la pronunciación y la corrección fonética en el aula de ELE». redELE Revista electrónica de didáctica / español lengua extranjera, 20, 5.

SANTos DíAZ, Inmaculada Clotilde; Trigo IbÁÑEZ, Ester y ROMERo OliVA, Manuel Francisco. 2020. "La activación del léxico disponible y su aplicación a la enseñanza de lenguas», Porta Linguarum, 33, 75-93. Recuperado el 27/07/2021, de http://hdl.handle.net/10481/62807

ŠIFRAR KALAN, Marjana. 2017. «Teaching pronunciation in Spanish/FL A1-B2 textbooks». Linguistica, 57(1), 313-330. https://doi.org/10.4312/linguistica.57.1.313330

SumnER, Meghan; KIM, Seung; KING, Ed y McGowAN, Kevin. (2014). «The socially weighted encoding of spoken words: A dual-route approach to speech perception». Frontiers in psychology, 4, 1015

TwOMEY, Katherine y CANGElosi, Angelo. 2020. «Heads, shoulders, knees and toes». En C. Rowland, A. Theakston y K. Twomey (eds.) Current Perspectives on Child Language Acquisition. Amsterdam:John Benjamins Publishing Company, 39-64. https://doi.org/10.1075/tilar.27.02two

UsÓ VICIEDO, Lidia. 2009. «Creencias de los profesores de español en formación sobre la pronunciación del español». MarcoELE. Revista de Didáctica del Español como Lengua Extranjera, 8.

VIDES VARGAS, Amanda María. 2013. Guía logopédica para la internalización de fonemas consonánticos y vocálicos con apoyo gestual. Tesis nivel técnico. Escuela de Ciencias Psicológicas, Guatemala.

VV. AA. 2008. «pronunciación». Diccionario de términos clave de ELE. Recuperado el 28/07/2021, de https://cvc.cervantes.es/ensenanza/biblioteca_ele/diccio_ele/diccionario/pronunciacion. htm 